\title{
Staggered Diquarks for Singly Heavy Baryons
}

\section{Steven Gottlieb}

Department of Physics, Indiana University, Bloomington, 47405, IN, U.S.A.

E-mail: sg@denali.physics.indiana.edu

\section{Heechang $\mathrm{Na}$}

Department of Physics, Indiana University, Bloomington, 47405, IN, U.S.A.

E-mail: heenadindiana.edux

\section{Kazuhiro Nagata*}

Department of Physics, Indiana University, Bloomington, 47405, IN, U.S.A.

E-mail: knagata@indiana.edu

In the staggered fermion formulation of lattice QCD, we construct diquark operators which are to be embedded in singly heavy baryons. The group theoretical connections between continuum and lattice staggered diquark representations are established.

The XXV International Symposium on Lattice Field Theory

July 30 - August 42007

Regensburg, Germany

\footnotetext{
* Speaker.
} 


\begin{tabular}{c|cccc||c}
\hline Baryon & $J^{P}$ & $Z$ & Content & $\left(S U(2)_{S}, S U(2)_{I}\right)_{Z}$ & $\left(S U(2)_{S}, S U(8)_{x, y}, S U(4)_{z}\right)_{Z}$ \\
\hline$\Lambda_{Q}$ & $\frac{1}{2}^{+}$ & 0 & $(l l) Q$ & $\left(\mathbf{1}_{\mathbf{A}}, \mathbf{1}_{\mathbf{A}}\right)_{0}$ & $\left(\mathbf{1}_{\mathbf{A}}, \mathbf{2 8}_{\mathbf{A}}, \mathbf{1}\right)_{0}$ \\
$\Xi_{Q}$ & $\frac{1}{2}^{+}$ & -1 & $(l s) Q$ & $\left(\mathbf{1}_{\mathbf{A}}, \mathbf{2}\right)_{-1}$ & $\left(\mathbf{1}_{\mathbf{A}}, \mathbf{8}, \mathbf{4}\right)_{-1}$ \\
$\Sigma_{Q}^{(*)}$ & $\frac{1}{2}^{+}\left(^{\frac{3}{2}}{ }^{+}\right)$ & 0 & $(l l) Q$ & $\left(\mathbf{3}_{\mathbf{S}}, \mathbf{3}_{\mathbf{S}}\right)_{0}$ & $\left(\mathbf{3}_{\mathbf{S}}, \mathbf{3 6} \mathbf{s}, \mathbf{1}\right)_{0}$ \\
$\Xi_{Q}^{\prime(*)}$ & $\frac{1}{2}^{+}\left(\frac{3}{2}^{+}\right)$ & -1 & $(l s) Q$ & $\left(\mathbf{3}_{\mathbf{S}}, \mathbf{2}\right)_{-1}$ & $\left(\mathbf{3}_{\mathbf{S}}, \mathbf{8}, \mathbf{4}\right)_{-1}$ \\
$\Omega_{Q}^{(*)}$ & $\frac{1}{2}^{+}\left(^{+} \frac{3}{2}^{+}\right)$ & -2 & $(s s) Q$ & $\left(\mathbf{3}_{\mathbf{S}}, \mathbf{1}\right)_{-2}$ & $\left(\mathbf{3}_{\mathbf{S}}, \mathbf{1}, \mathbf{1 0} \mathbf{1}_{\mathbf{S}}\right)_{-2}$ \\
\hline
\end{tabular}

Table 1: Quantum numbers for singly heavy baryons for $2+1$ flavor symmetry : $Z$ denotes strangeness. The states with asterisks represent the spin $\frac{3}{2}$ states. The fifth and sixth column represent the light diquark irreps for single taste and four tastes, respectively.

\section{Introduction}

There have been a number of attempts to investigate heavy baryons in terms of experimental as well as theoretical methods. In lattice QCD, several calculations have been performed in the quenched regime [1, 2, 3, 4, 5, 6, 7] and given a fair agreement with the experimental results. In [8], two of the authors have reported the results of preliminary study for the singly charmed baryon mass spectrum using the data of $2+1$ flavors dynamical improved staggered quarks. Since the staggered fermion provides very fast simulations and much less statistical errors compared to the other available frameworks, (see, for example, Ref [9]), it is worthwhile to pursue more extensive studies of those baryons in terms of staggered light quarks. It is then desired to establish the group theoretical classification of pairs of staggered quarks (staggered diquarks) in order to extract the desired spin and parity state in the continuum limit. In [10], we construct all the possible timelocal staggered diquarks embedded in singly heavy baryons and establish the group theoretical connections between lattice operators and continuum representations w.r.t. spin, taste and $2+1$ flavor symmetries.

\section{Staggered Diquarks in the Continuum Spacetime}

A singly heavy baryon operator consists of two light quarks (up, down or strange) and one heavy quark (charm or bottom (or top)). The quantum numbers of singly heavy baryons are listed in Table 1. In this section, we classify the irreps of the staggered diquarks w.r.t. spin, flavor and taste symmetry group in the continuum spacetime. We especially take $2+1$ as the flavor symmetry group under which the recent dynamical simulations of staggered lattice QCD are performed.

Let us begin with reviewing the diquark irreps for mass degenerate light quarks of single taste, namely physical valence quarks. The success of non-relativistic $S U(6)$ quark model suggests that the diquarks should belong to the irreps $\mathbf{2 1}_{S}$, the symmetric part of $\mathbf{6} \otimes \mathbf{6}$, which has the following decomposition into $S U(2)_{S} \times S U(3)_{F}$, the direct product of non-relativistic spin and $S U(3)$ flavor,

$$
\begin{aligned}
S U(6) & \supset S U(2)_{S} \times S U(3)_{F} \\
\mathbf{2 1}_{\mathbf{S}} & \rightarrow\left(\mathbf{3}_{\mathbf{S}}, \mathbf{6}_{\mathbf{S}}\right) \oplus\left(\mathbf{1}_{\mathbf{A}}, \mathbf{3}_{\mathbf{A}}\right),
\end{aligned}
$$


where the labeling represents the dimension of each irreps while the subscripts $\mathbf{S}$ and ${ }_{\mathbf{A}}$ indicate the symmetric and anti-symmetric part, respectively. For $2+1$ flavors, $S U(3)_{F}$ is decomposed into $S U(2)_{I}$ isospin group. Accordingly, we have

$$
\begin{aligned}
S U(2)_{S} \times S U(3)_{F} & \supset S U(2)_{S} \times S U(2)_{I} \\
\left(\mathbf{3}_{\mathbf{S}}, \mathbf{6}_{\mathbf{S}}\right) & \rightarrow\left(\mathbf{3}_{\mathbf{S}}, \mathbf{3}_{\mathbf{S}}\right)_{0} \oplus\left(\mathbf{3}_{\mathbf{S}}, \mathbf{2}\right)_{-1} \oplus\left(\mathbf{3}_{\mathbf{S}}, \mathbf{1}\right)_{-2} \\
\left(\mathbf{1}_{\mathbf{A}}, \mathbf{3}_{\mathbf{A}}\right) & \rightarrow\left(\mathbf{1}_{\mathbf{A}}, \mathbf{1}_{\mathbf{A}}\right)_{0} \oplus\left(\mathbf{1}_{\mathbf{A}}, \mathbf{2}\right)_{-1},
\end{aligned}
$$

where subscripts $0,-1,-2$ denote the strangeness associated with each irrep. Each of these irreps has one-to-one correspondence to the physical diquark state in singly heavy baryons, as listed in the second last column of Table 1 .

As for the light staggered quarks having four tastes with degenerate mass, the above $S U(3)_{F}$ flavor symmetry is extended to $S U(12)_{f}$ flavor-taste symmetry [11]. Correspondingly, the staggered diquarks belong to the symmetric irreps of $S U(24)$ which has the following decomposition,

$$
\begin{aligned}
S U(24) & \supset S U(2)_{S} \times S U(12)_{f} \\
\mathbf{3 0 0}_{\mathbf{S}} & \rightarrow\left(\mathbf{3}_{\mathbf{S}}, \mathbf{7 8}_{\mathbf{S}}\right) \oplus\left(\mathbf{1}_{\mathbf{A}}, \mathbf{6 6}_{\mathbf{A}}\right) .
\end{aligned}
$$

For $2+1$ flavor staggered quarks, the $S U(12)_{f}$ flavor-taste symmetry group is broken to $S U(8)_{x, y} \times$ $S U(4)_{z}$, where $S U(8)_{x, y}$ denotes the symmetry group for two light valence quarks while $S U(4)_{z}$ the one for a strange valence quark. The decomposition of $S U(12)_{f}$ into $S U(8)_{x y} \times S U(4)_{z}$ gives,

$$
\begin{aligned}
S U(2)_{S} \times S U(12)_{f} & \supset S U(2)_{S} \times S U(8)_{x, y} \times S U(4)_{z} \\
\left(\mathbf{3}_{\mathbf{S}}, \mathbf{7 8}_{\mathbf{S}}\right) & \rightarrow\left(\mathbf{3}_{\mathbf{S}}, \mathbf{3 6} \mathbf{s}, \mathbf{1}\right)_{0} \oplus\left(\mathbf{3}_{\mathbf{S}}, \mathbf{8}, \mathbf{4}\right)_{-1} \oplus\left(\mathbf{3}_{\mathbf{S}}, \mathbf{1}, \mathbf{1 0}_{\mathbf{S}}\right)_{-2}, \\
\left(\mathbf{1}_{\mathbf{A}}, \mathbf{6 6}_{\mathbf{A}}\right) & \rightarrow\left(\mathbf{1}_{\mathbf{A}}, \mathbf{2 8}_{\mathbf{A}}, \mathbf{1}\right)_{0} \oplus\left(\mathbf{1}_{\mathbf{A}}, \mathbf{8}, \mathbf{4}\right)_{-1} \oplus\left(\mathbf{1}_{\mathbf{A}}, \mathbf{1}, \mathbf{6}_{A}\right)_{-2},
\end{aligned}
$$

We assume in the continuum limit that the taste symmetry restores and all the four tastes become equivalent. Then we see all the irreps except $\left(\mathbf{1}_{\mathbf{A}}, \mathbf{1}, \mathbf{6}_{\mathbf{A}}\right)_{-2}$ are to be degenerate with physical diquarks under this assumption. We list the staggered irreps for the physical diquarks in the last column of Table 1 . The strangeness -2 spin singlet diquark state $\left(\mathbf{1}_{\mathbf{A}}, \mathbf{1}, \mathbf{6}_{\mathbf{A}}\right)_{-2}$ in $(2.6)$ does not correspond to any physical state in continuum limit.

In order to make contact with the lattice symmetry group, we further decompose the physical states into $S U(2)_{S} \times S U(2)_{I} \times S U(4)_{T}$ as follows,

$$
\begin{aligned}
& S U(2)_{S} \times S U(8)_{x, y} \times S U(4)_{z} \supset S U(2)_{S} \times S U(2)_{I} \times S U(4)_{T} \\
& \Sigma_{Q}^{(*)}: \quad\left(\mathbf{3}_{\mathbf{S}}, \mathbf{3 6}_{\mathbf{S}}, \mathbf{1}\right)_{0} \rightarrow\left(\mathbf{3}_{\mathbf{S}}, \mathbf{3}_{\mathbf{S}}, \mathbf{1 0}_{\mathbf{S}}\right)_{0} \oplus\left(\mathbf{3}_{\mathbf{S}}, \mathbf{1}_{\mathbf{A}}, \mathbf{6}_{\mathbf{A}}\right)_{0} \\
& \Xi_{Q}^{\prime(*)}: \quad\left(\mathbf{3}_{\mathbf{S}}, \mathbf{8}, \mathbf{4}\right)_{-1} \rightarrow\left(\mathbf{3}_{\mathrm{S}}, \mathbf{2}, \mathbf{1 0} \mathbf{S}_{\mathrm{S}}\right)_{-1} \oplus\left(\mathbf{3}_{\mathrm{S}}, \mathbf{2}, \mathbf{6}_{\mathbf{A}}\right)_{-1} \\
& \Omega_{Q}^{(*)}:\left(\mathbf{3}_{\mathbf{S}}, \mathbf{1}, \mathbf{1 0}_{\mathbf{S}}\right)_{-2} \rightarrow\left(\mathbf{3}_{\mathbf{S}}, \mathbf{1}, \mathbf{1 0}_{\mathbf{S}}\right)_{-2} \\
& \Lambda_{Q}:\left(\mathbf{1}_{\mathbf{A}}, \mathbf{2 8}_{\mathbf{A}}, \mathbf{1}\right)_{0} \rightarrow\left(\mathbf{1}_{\mathbf{A}}, \mathbf{1}_{\mathbf{A}}, \mathbf{1 0}_{\mathbf{S}}\right)_{0} \oplus\left(\mathbf{1}_{\mathbf{A}}, \mathbf{3}_{\mathbf{S}}, \mathbf{6}_{\mathbf{A}}\right)_{0} \\
& \Xi_{Q}: \quad\left(\mathbf{1}_{\mathbf{A}}, \mathbf{8}, \mathbf{4}\right)_{-1} \rightarrow\left(\mathbf{1}_{\mathbf{A}}, \mathbf{2}, \mathbf{1 0}_{\mathbf{S}}\right)_{-1} \oplus\left(\mathbf{1}_{\mathbf{A}}, \mathbf{2}, \mathbf{6}_{\mathbf{A}}\right)_{-1} .
\end{aligned}
$$

The main goal of this article is to construct the lattice staggered diquark operators categorized into the physical irreps given in the right hand sides of (2.7)-(2.11). 


\section{Staggered Diquarks on the Lattice}

The symmetry group of staggered fermion action on Euclidean lattice was first elaborated in $[15,16]$ and successively applied to classifying staggered baryons as well as mesons [12, 13, 14]. The important symmetries of staggered fermions in our study are $90^{\circ}$ rotations $R^{(\rho \sigma)}$, shift transformations $S_{\mu}$, space inversion $I_{s}$. Since the shift operations $S_{\mu}$ contain taste matrices, pure translations $T_{\mu}$ may be represented by the square of $S_{\mu}, T_{\mu}=S_{\mu}^{2}$. Discrete taste transformations in Hilbert space are readily defined by $\Xi_{\mu} \equiv S_{\mu} T_{\mu}^{-\frac{1}{2}}$. The $\Xi_{\mu}$ generate 32 element Clifford group which is isomorphic to the discrete subgroup of $S U(4)_{T}$ in the continuum spacetime. Since the space inversion contains a taste transformation $\Xi_{4}$, the parity should be defined by $P=\Xi_{4} I_{s}$. Note that the parity is non-locally defined in time direction since $\Xi_{4}$ is non-local in time. For the purpose of spectroscopy, we are particularly interested in a symmetry group generated by the transformations which are local in time and commuting with $T_{4}$. Such a group is called geometrical time slice group $(G T S)$ which is given by

$$
G T S=G\left(R^{(k l)}, \Xi_{m}, I_{S}\right),
$$

where $k, l, m=1 \sim 3[12,13,14]$. The defining representation of GTS is given by the staggered quark fields projected on zero spatial momentum. It is an eight dimensional representation denoted as $\mathbf{8}$. The anti-staggered quark fields also belong to the representation $\mathbf{8}$. The GTS representation of staggered diquark is accordingly expressed by $\mathbf{8} \times \mathbf{8}$. The decomposition of $\mathbf{8} \times \mathbf{8}$ into the bosonic irreps is given in [13],

$$
\mathbf{8} \times \mathbf{8}=\sum_{\sigma_{s}= \pm 1, \sigma_{123}= \pm 1}\left\{\mathbf{1}^{\sigma_{s} \sigma_{123}}+\mathbf{3}^{\sigma_{s} \sigma_{123}}+\mathbf{3}^{\prime \prime \sigma_{s} \sigma_{123}}+\mathbf{3}^{\prime \prime \prime \prime} \sigma_{s} \sigma_{123}+\boldsymbol{6}^{\sigma_{s} \sigma_{123}}\right\},
$$

where $1,3,3^{\prime \prime}, 3^{\prime \prime \prime \prime}$ and $\mathbf{6}$ are representing the bosonic representations of GTS with $\sigma_{s}$, the eigenvalue of $I_{s}$ and $\sigma_{123}$, the eigenvalue of $D\left(\Xi_{1} \Xi_{2} \Xi_{3}\right)$.

The irreducibly transforming diquark operators are listed in Table 2 and 3 . As in the meson case, all the irreps are categorized into four classes from 0 to 3, depending on how far the two staggered quarks are displaced each other. The third column of the tables gives the operator form of the diquarks. The fourth column gives the corresponding GTS irreps. The $\eta_{\mu}$ and $\zeta_{\mu}$ denote the sign factors defined by $\eta_{\mu}(x)=(-1)^{x_{1}+\cdots+x_{\mu-1}}$ and $\zeta_{\mu}(x)=(-1)^{x_{\mu+1}+\cdots+x_{4}}$, respectively, while $\varepsilon$ is defined as $\varepsilon(x)=(-1)^{x_{1}+x_{2}+x_{3}+x_{4}}$. The $D_{k}$ represents the symmetric shift operators defined by $D_{k} \phi(\mathbf{x})=\frac{1}{2}\left[\phi\left(\mathbf{x}+\mathbf{a}_{k}\right)+\phi\left(\mathbf{x}-\mathbf{a}_{k}\right)\right]$. For notational simplicity, the sum over $\mathbf{x}$, the color and flavor indices are suppressed without any confusion. For example, $\chi \eta_{k} D_{k} \chi$ stands for $\sum_{\mathbf{x}} \chi_{f_{1}}^{a}(\mathbf{x}, t) \eta_{k}(x) D_{k} \chi_{f_{2}}^{b}(\mathbf{x}, t)$. As far as the lattice symmetry group GTS is concerned, each diquark operator is formally corresponding to the meson operator given in [13] through replacing the leftmost $\chi$ by $\bar{\chi}$. This is because the staggered quark and anti-quark belong to the same GTS irrep for each color and flavor. The $\sigma_{4}$ in the fifth column denotes the eigenvalue of $X_{4}$ with which the parity is given by $P=\sigma_{s} \sigma_{4}$. The sixth column gives the spin and taste matrices $\Gamma_{S} \otimes \Gamma_{T}$ which come into the diquark operators in the spin-taste basis, $\psi^{T}\left(C \Gamma_{S} \otimes\left(\Gamma_{T} C^{-1}\right)^{T}\right) \psi$, where the superscript $T$ denotes transpose and $C$ denotes the charge conjugation matrix. The presence of $C$ and $C^{-1}$ ensures the covariant properties under the spin and taste rotations in the continuum limit. Notice that the assignment of $\Gamma_{S} \otimes \Gamma_{T}$ for each GTS irrep is systematically different from the meson case, where the operators are given by $\bar{\psi}\left(\Gamma_{S} \otimes\left(\Gamma_{T}\right)^{T}\right) \psi$ in the spin-taste basis. 


\begin{tabular}{|c|c|c|c|c|c|c|c|c|}
\hline class & No. & operator & $G T S\left(\overline{\mathbf{r}}^{\sigma_{s} \sigma_{123}}\right)$ & $\sigma_{4}$ & $\Gamma_{S} \otimes \Gamma_{T}$ & $J^{P}$ & order & $\left(S U(2)_{S}, S U(4)_{T}\right)$ \\
\hline \multirow[t]{8}{*}{0} & 1 & $\chi \chi$ & $\mathbf{1}^{++}$ & + & $\gamma_{5} \otimes \gamma_{5}$ & $0^{+}$ & 1 & $\left(\mathbf{1}_{\mathrm{A}}, \mathbf{6}_{\mathrm{A}}\right)$ \\
\hline & & & & - & $\gamma_{4} \otimes \gamma_{4}$ & $0^{-}$ & $p / E$ & \\
\hline & 2 & $\eta_{4} \zeta_{4} \chi \chi$ & $\mathbf{1}^{+-}$ & + & $\gamma_{4} \gamma_{5} \otimes \gamma_{4} \gamma_{5}$ & $0^{+}$ & 1 & $\left(\mathbf{1}_{\mathbf{A}}, \mathbf{6}_{\mathbf{A}}\right)$ \\
\hline & & & & - & $1 \otimes 1$ & $0^{-}$ & $p / E$ & \\
\hline & 3 & $\eta_{k} \varepsilon \zeta_{k} \chi \chi$ & $3^{\prime \prime \prime \prime}+-$ & + & $\gamma_{k} \otimes \gamma_{k}$ & $1^{+}$ & 1 & $\left(\mathbf{3}_{\mathrm{S}}, \mathbf{1 0}_{\mathrm{S}}\right)$ \\
\hline & & & & - & $\gamma_{l} \gamma_{m} \otimes \gamma_{l} \gamma_{m}$ & $1^{-}$ & $p / E$ & \\
\hline & 4 & $\eta_{4} \zeta_{4} \eta_{k} \varepsilon \zeta_{k} \chi \chi$ & $\mathbf{3}^{\prime \prime \prime \prime}++$ & + & $\gamma_{k} \gamma_{4} \otimes \gamma_{k} \gamma_{4}$ & $1^{+}$ & 1 & $\left(\mathbf{3}_{\mathrm{S}}, 10_{\mathrm{S}}\right)$ \\
\hline & & & & - & $\gamma_{k} \gamma_{5} \otimes \gamma_{k} \gamma_{5}$ & $1^{-}$ & $p / E$ & \\
\hline \multirow[t]{12}{*}{1} & 5 & $\chi \eta_{k} D_{k} \chi$ & $\mathbf{3}^{-+}$ & + & $\gamma_{k} \gamma_{5} \otimes \gamma_{5}$ & $1^{-}$ & $p / E$ & \\
\hline & & & & - & $\gamma_{k} \gamma_{4} \otimes \gamma_{4}$ & $1^{+}$ & 1 & $\left(\mathbf{3}_{\mathrm{S}}, \mathbf{1 0}_{\mathrm{S}}\right)$ \\
\hline & 6 & $\eta_{4} \zeta_{4} \chi \eta_{k} D_{k} \chi$ & $3^{--}$ & + & $\gamma_{l} \gamma_{m} \otimes \gamma_{4} \gamma_{5}$ & $1^{-}$ & $p / E$ & \\
\hline & & & & - & $\gamma_{k} \otimes 1$ & $1^{+}$ & 1 & $\left(3_{S}, 6_{A}\right)$ \\
\hline & 7 & $\chi \varepsilon \zeta_{k} D_{k} \chi$ & $\mathbf{3}^{\prime \prime--}$ & + & $1 \otimes \gamma_{k}$ & $0^{-}$ & $p / E$ & \\
\hline & & & & - & $\gamma_{4} \gamma_{5} \otimes \gamma_{l} \gamma_{m}$ & $0^{+}$ & 1 & $\left(\mathbf{1}_{\mathbf{A}}, \mathbf{1 0}_{\mathbf{S}}\right)$ \\
\hline & 8 & $\eta_{4} \zeta_{4} \chi \varepsilon \zeta_{k} D_{k} \chi$ & $\mathbf{3}^{\prime \prime-+}$ & + & $\gamma_{4} \otimes \gamma_{k} \gamma_{4}$ & $0^{-}$ & $p / E$ & \\
\hline & & & & - & $\gamma_{5} \otimes \gamma_{k} \gamma_{5}$ & $0^{+}$ & 1 & $\left(\mathbf{1}_{\mathrm{A}}, \mathbf{6}_{\mathrm{A}}\right)$ \\
\hline & 9 & $\eta_{k} \varepsilon \zeta_{k} \chi \eta_{l} D_{l} \chi$ & $6^{--}$ & + & $\gamma_{k} \gamma_{l} \otimes \gamma_{k}$ & $1^{-}$ & $p / E$ & \\
\hline & & & & - & $\gamma_{m} \otimes \gamma_{l} \gamma_{m}$ & $1^{+}$ & 1 & $\left(\mathbf{3}_{\mathrm{S}}, \mathbf{1 0}_{\mathrm{S}}\right)$ \\
\hline & 10 & $\eta_{4} \zeta_{4} \eta_{k} \varepsilon \zeta_{k} \chi \eta_{l} D_{l} \chi$ & $6^{-+}$ & + & $\gamma_{m} \gamma_{5} \otimes \gamma_{k} \gamma_{4}$ & $1^{-}$ & $p / E$ & \\
\hline & & & & - & $\gamma_{m} \gamma_{4} \otimes \gamma_{k} \gamma_{5}$ & $1^{+}$ & 1 & $\left(3_{S}, 6_{A}\right)$ \\
\hline
\end{tabular}

Table 2: GTS irrep., $\sigma_{4}, \Gamma_{S} \otimes \Gamma_{T}$ and continuum states for staggered diquark operators up to class 1 . $(k, l, m=1 \sim 3, k \neq l \neq m \neq k)$. The summation over $\mathbf{x}$, flavor and color indices are omitted.

\begin{tabular}{|c|c|c|c|c|c|c|c|c|}
\hline class & No. & operator & $G T S\left(\overline{\mathbf{r}}^{\sigma_{s} \sigma_{123}}\right)$ & $\sigma_{4}$ & $\overline{\Gamma_{S} \otimes \Gamma_{T}}$ & $J^{P}$ & order & $\left(S U(2)_{S}, S U(4)_{T}\right)$ \\
\hline \multirow[t]{12}{*}{2} & 11 & $\chi \eta_{k} D_{k}\left\{\eta_{l} D_{l} \chi\right\}$ & $\mathbf{3}^{++}$ & + & $\gamma_{m} \gamma_{4} \otimes \gamma_{5}$ & $1^{+}$ & 1 & $\left(3_{S}, 6_{A}\right)$ \\
\hline & & & & - & $\gamma_{m} \gamma_{5} \otimes \gamma_{4}$ & $1^{-}$ & $p / E$ & \\
\hline & 12 & $\eta_{4} \zeta_{4} \chi \eta_{k} D_{k}\left\{\eta_{l} D_{l} \chi\right\}$ & $3^{+-}$ & + & $\gamma_{m} \otimes \gamma_{4} \gamma_{5}$ & $1^{+}$ & 1 & $\left(3_{S}, 6_{A}\right)$ \\
\hline & & & & - & $\gamma_{k} \gamma_{l} \otimes 1$ & $1^{-}$ & $p / E$ & \\
\hline & 13 & $\chi \zeta_{k} D_{k}\left\{\zeta_{l} D_{l} \chi\right\}$ & $3^{\prime \prime++}$ & + & $\gamma_{5} \otimes \gamma_{m} \gamma_{4}$ & $0^{+}$ & 1 & $\left(\mathbf{1}_{\mathrm{A}}, \mathbf{1 0}_{\mathrm{S}}\right)$ \\
\hline & & & & - & $\gamma_{4} \otimes \gamma_{m} \gamma_{5}$ & $0^{-}$ & $p / E$ & \\
\hline & 14 & $\eta_{4} \zeta_{4} \chi \zeta_{k} D_{k}\left\{\zeta_{l} D_{l} \chi\right\}$ & $3^{\prime \prime+-}$ & + & $\gamma_{4} \gamma_{5} \otimes \gamma_{m}$ & $0^{+}$ & 1 & $\left(\mathbf{1}_{A}, \mathbf{1 0}_{S}\right)$ \\
\hline & & & & - & $1 \otimes \gamma_{k} \gamma_{l}$ & $0^{-}$ & $p / E$ & \\
\hline & 15 & $\eta_{m} \zeta_{m} \chi \eta_{k} D_{k}\left\{\zeta_{l} D_{l} \chi\right\}$ & $6^{++}$ & + & $\gamma_{l} \gamma_{4} \otimes \gamma_{k} \gamma_{4}$ & $1^{+}$ & 1 & $\left(\mathbf{3}_{\mathrm{S}}, \mathbf{1 0}_{\mathrm{S}}\right)$ \\
\hline & & & & - & $\gamma_{l} \gamma_{5} \otimes \gamma_{k} \gamma_{5}$ & $1^{-}$ & $p / E$ & \\
\hline & 16 & $\eta_{4} \zeta_{4} \eta_{m} \zeta_{m} \chi \eta_{k} D_{k}\left\{\zeta_{l} D_{l} \chi\right\}$ & $6^{+-}$ & + & $\gamma_{l} \otimes \gamma_{k}$ & $1^{+}$ & 1 & $\left(\mathbf{3}_{\mathrm{S}}, 10_{\mathrm{S}}\right)$ \\
\hline & & & & - & $\gamma_{k} \gamma_{m} \otimes \gamma_{l} \gamma_{m}$ & $1^{-}$ & $p / E$ & \\
\hline \multirow[t]{8}{*}{3} & 17 & $\chi \eta_{1} D_{1}\left\{\eta_{2} D_{2}\left\{\eta_{3} D_{3} \chi\right\}\right\}$ & $\mathbf{1}^{-+}$ & + & $\gamma_{4} \otimes \gamma_{5}$ & $0^{-}$ & $p / E$ & \\
\hline & & & & - & $\gamma_{5} \otimes \gamma_{4}$ & $0^{+}$ & 1 & $\left(\mathbf{1}_{\mathrm{A}}, \mathbf{1 0}_{\mathrm{S}}\right)$ \\
\hline & 18 & $\eta_{4} \zeta_{4} \chi \eta_{1} D_{1}\left\{\eta_{2} D_{2}\left\{\eta_{3} D_{3} \chi\right\}\right\}$ & $\mathbf{1}^{--}$ & + & $1 \otimes \gamma_{4} \gamma_{5}$ & $0^{-}$ & $p / E$ & \\
\hline & & & & - & $\gamma_{4} \gamma_{5} \otimes 1$ & $0^{+}$ & 1 & $\left(\mathbf{1}_{\mathrm{A}}, \mathbf{6}_{\mathrm{A}}\right)$ \\
\hline & 19 & $\eta_{k} \varepsilon \zeta_{k} \chi \eta_{1} D_{1}\left\{\eta_{2} D_{2}\left\{\eta_{3} D_{3} \chi\right\}\right\}$ & $3^{\prime \prime \prime \prime--}$ & + & $\gamma_{l} \gamma_{m} \otimes \gamma_{k}$ & $1^{-}$ & $p / E$ & \\
\hline & & & & - & $\gamma_{k} \otimes \gamma_{l} \gamma_{m}$ & $1^{+}$ & 1 & $\left(\mathbf{3}_{S}, \mathbf{1 0}_{S}\right)$ \\
\hline & 20 & $\eta_{4} \zeta_{4} \eta_{k} \varepsilon \zeta_{k} \chi \eta_{1} D_{1}\left\{\eta_{2} D_{2}\left\{\eta_{3} D_{3} \chi\right\}\right\}$ & $3^{\prime \prime \prime \prime-+}$ & + & $\gamma_{k} \gamma_{5} \otimes \gamma_{k} \gamma_{4}$ & $1^{-}$ & $p / E$ & \\
\hline & & & & - & $\gamma_{k} \gamma_{4} \otimes \gamma_{k} \gamma_{5}$ & $1^{+}$ & 1 & $\left(\mathbf{3}_{\mathrm{S}}, \mathbf{6}_{\mathrm{A}}\right)$ \\
\hline
\end{tabular}

Table 3: GTS irrep., $\sigma_{4}, \Gamma_{S} \otimes \Gamma_{T}$ and continuum states for staggered diquark operators class 2 and 3 . $(k, l, m=1 \sim 3, k \neq l \neq m \neq k)$. The summation over $\mathbf{x}$, flavor and color indices are omitted. 


\section{Connection between lattice and continuum irreps}

Consulting the relations between lattice $\overline{R F}$ irreps and continuum spin irreps given in [13] and assuming that the ground states of lattice irreps correspond to the lowest possible spin in the continuum limit, one could make an assignment of spin and parity $J^{P}$ for each GTS irrep. See the seventh column of Tables 2 and 3 . One also see that the combinations, $C \Gamma_{S}=C \gamma_{k}, C \gamma_{k} \gamma_{4}, C \gamma_{4} \gamma_{5}, C \gamma_{5}$, generate upper $\times$ upper products of the Dirac spinors in Dirac representation for each taste and then give rise to $\mathscr{O}(1)$ contributions, while the combinations, $C \Gamma_{S}=C, C \gamma_{4}, C \gamma_{k} \gamma_{l}, C \gamma_{k} \gamma_{5}$, generate upper $\times$ lower products, so that they are suppressed by $\mathscr{O}(p / E)$ in the non-relativistic limit. See the second last column of Table 2 and 3 . An important notice here is that only the positive parity states survive in the non-relativistic limit, which is in accordance with the property of physical diquarks. As for the $S U(4)_{T}$ irreps, one sees that the combinations, $\Gamma_{T} C^{-1}=\gamma_{k} C^{-1}, \gamma_{4} C^{-1}, \gamma_{k} \gamma_{l} C^{-1}, \gamma_{k} \gamma_{4} C^{-1}$, are symmetric so that they altogether belong to $\mathbf{1 0}_{\mathrm{S}}$ irrep of $S U(4)_{T}$, while the anti-symmetric six combinations $\Gamma_{T} C^{-1}=C^{-1}, \gamma_{k} \gamma_{5} C^{-1}, \gamma_{4} \gamma_{5} C^{-1}, \gamma_{5} C^{-1}$, belong to $\mathbf{6}_{\mathrm{A}}$ irrep of $S U(4)_{T}$. The assignments of non-relativistic $S U(2)_{S} \times S U(4)_{T}$ for the lattice irreps are readily given for the $\mathscr{O}(1)$ operators. They are listed in the last column of the tables. The final step is to take into account the $2+1$ flavor symmetry, which could be done in a straightforward manner. The decomposition of continuum spin, $2+1$ flavor and taste symmetry group into the lattice symmetry group is given by,

$$
S U(2)_{S} \times S U(2)_{I} \times S U(4)_{T} \supset S U(2)_{I} \times G T S .
$$

In Table 4, we list all the lattice diquark operators which are local in time and categorize them into each continuum irrep $\left(S U(2)_{S}, S U(2)_{I}, S U(4)_{T}\right)_{Z}$ previously given in (2.7)-(2.11).

\section{Summary}

Continuum and lattice irreps of staggered diquarks with $S U(4)$ taste symmetry in $2+1$ flavors were studied. We have started from the $S U(24)$ symmetry group which is the $S U(4)$ taste extension of ordinary $S U(6)$ non-relativistic quark model. This procedure has been also taken in the study of staggered baryon classifications [11]. As for the lattice representations, we have consulted the lattice symmetry group of staggered fermion action elaborated in [15, 16]. Although the irreps of lattice symmetry group GTS cannot have any definite parity, we have explicitly shown that only the positive parity state contributes in the non-relativistic limit, which is in accordance with the property of physical diquarks.

\section{Acknowledgments}

We would like to thank S. Basak, C. Bernard and C. DeTar for useful discussions and comments. We thank J. Bailey for important comments on the physical states. This work has been supported by U.S. Department of Energy, Grant No. FG02-91ER 40661.

\section{References}

[1] K.C. Bowler et al. (UKQCD Collaboration), Phys. Rev. D54 (1996) 3619. 


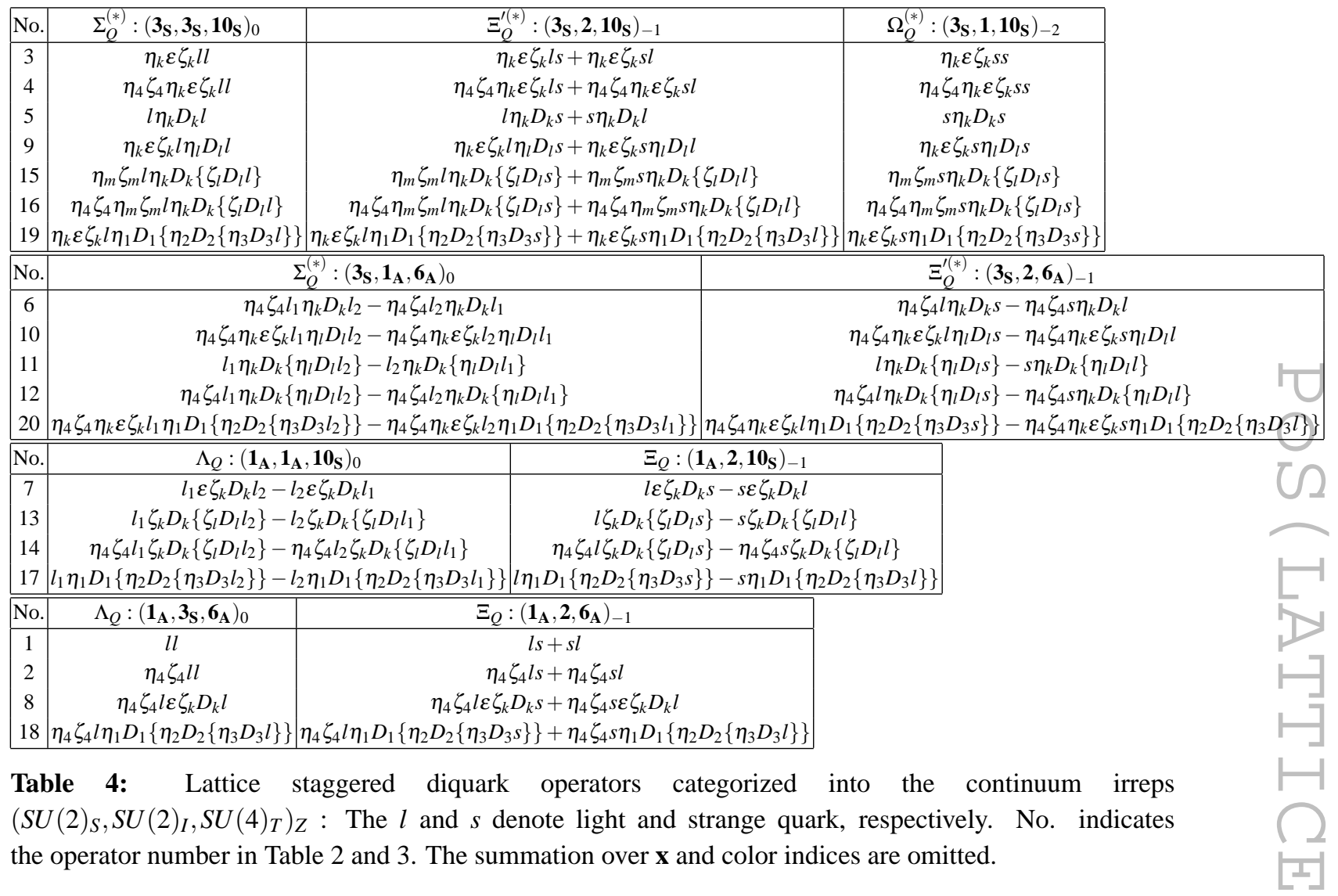

[2] J. M. Flynn, F. Mescia and A. S. B. Tariq (UKQCD Collaboration), JHEP 0307, 066 (2003)

[3] N. Mathur et al., Phys. Rev. D66 (2002) 014502.

[4] R.M. Woloshyn, Phys. Lett. B476 (2000) 309.

[5] S. Gottlieb and S. Tamhankar, Nucl. Phys. Proc. Suppl. 119 (2003) 644.

[6] A. Ali Khan et al., Phys. Rev. D62 (2000) 054505.

[7] T.W. Chiu and T.H.Hsieh, Nucl. Phys. A755 (2005) 471c.

[8] S. Gottlieb and H. Na, PoS LAT2006, 191 (2006) [hep-lat/ 0610009 ].

[9] C. Aubin et al.(MILC Collaboration), Phys. Rev. D70 (2004) 114501.

[10] S. Gottlieb, H. Na and K. Nagata, arXiv:0707.3537 [hep-lat].

[11] J. Bailey, Phys. Rev. D75, 114505 (2007).

[12] M.F.L. Golterman and J. Smit, Nucl. Phys. B255 (1985) 328.

[13] M.F.L. Golterman, Nucl. Phys. B273 (1986) 663.

[14] J. Smit, "Hadron Operators for Staggered Fermions", at Conf. Advances in Lattice Gauge Theory, Tallahassee, Fla., Apr 10-13, 1985.

[15] C.P. van den Doel and J. Smit, Nucl. Phys. B228 (1983) 122.

[16] M.F.L. Golterman and J. Smit, Nucl. Phys. B245 (1984) 61.

[17] S. Gupta, Phys. Rev. D60 (1999) 094505. 